\title{
SIGNIFICATION OF DEPTH OF PENETRATION IN EDDY CURRENT NON-DESTRUCTIVE TESTING
}

Eddy currents are utilized for non-destructive testing of conductive materials. The depth of penetration is a very important parameter as it defines the propagation of the electromagnetic wave as well as of the electromagnetic energy in conductive materials. Limitations of the nondestructive testing using eddy currents are therefore given by the parameter. The paper highlights the signification of the depth of penetration in eddy current non-destructive testing using numerical simulations. A tubular specimen with a defect of a variable depth is inspected using a bobbin type probe and the crack signals are calculated for the purpose. The finite element method is used for the numerical study.

\section{Introduction}

Non-destructive testing (NDT) is utilized to examine structural components because of localization and characterization of material properties' degradation (i.e. crack) that might cause malfunction of a component (e.g. reactors to fail, trains to derail, pipelines to burst, etc.) with economical and ecological impacts. The NDT is performed to assure consequent faultless operation of an inspected object without any mechanical damage. Recently, NDT methods are used not only for localization of a crack, but also for characterization of its size, shape, and orientation [1].

The number of NDT methods continuously grows as there are increasing demands for the inspection of structural components in different applications. However, six major NDT methods are mostly utilized, namely penetrant testing, magnetic particle testing, electromagnetic or eddy current testing (ECT), radiography, and ultrasonic testing.

This paper concentrates on the eddy current non-destructive testing. Numerical simulations of electromagnetic field distribution using the finite element method are done to simulate inspection of a tubular specimen with a defect of a variable depth. A bobbin type probe is used for the testing. Different frequencies of an excitation current are considered to highlight the signification of the depth of penetration in eddy current non-destructive testing.

\section{Eddy current non-destructive testing}

An inductance coil driven by an alternating current generates an alternating electromagnetic field [1]. When a conductive material is placed in proximity of the coil, the electromotive force is induced in the material and eddy currents (EC) flow there according to the electromotive force. The primary, exciting, electromagnetic field produced by the exciting coil is attenuated by a secondary, reaction, magnetic field generated by eddy currents. If the flow pattern of eddy currents is changed by a presence of an anomaly, it influences the reaction field and, therefore, also the exciting electromagnetic field. This change can be measured as a variation in the coil impedance. In a case of a transmitter - receiver probe, the field fluctuation is sensed through the change of induced voltage in a pick-up coil. Exact location of a defect can be evaluated by tracking of the measured signal when the probe is moved over the surface of a material.

There are several possible configurations of eddy current testing probes. The probe consists of at least one exciting coil and one detecting coil to pick-up the signal. Although, only one coil is used for both purposes in absolute probe [2]. The design of the probe has to allow surface testing of an examined material. The measured values of the impedance or the induced voltage are gathered to a computer or they are displayed and visually evaluated on a scope. It is common to plot the eddy current signals in the complex plane as not only the amplitude but also the phase of the signal carries important information about any change in material properties.

One of the major advantages of ECT is the variety of inspections and measurements that can be performed. ECT can be used for:

- crack detection,

- material thickness measurements,

- coating thickness measurements,

- conductivity measurements [3].

ECT is sensitive to small cracks and it is used for detection of surface and near surface defects. There is no need for physical contact between the probe and an inspected material and the inspection gives immediate results. Despite all the mentioned advantages there are several drawbacks. Only conductive materials can be inspected while the volumetric inspection is limited by the depth of penetration.

\footnotetext{
* Tomáś Marek

Department of Electromagnetic and Biomedical Engineering, Faculty of Electrical Engineering, University of Žilina, Univerzitná 1, 01026 Žilina, Eemail: marek@fel.utc.sk
} 


\section{Electromagnetic waves propagation and the depth of penetration}

The theory of quasi-stationary electromagnetic field is used for theoretical description of eddy currents in conductive media. The simplified Maxwell equations [4], [5] well define the problem as the displacement current $\partial \mathbf{D} / \partial t$ is eliminated from the equations because conductive currents are dominant in ECT as the problem is solved in conductive materials.

Propagation of the electromagnetic waves in the air and in the material can be described by wave equations [4]. It is possible to write a wave equation for any properly defined vector or scalar function (e.g. E, H, B, D, A, V, etc.) in general [4]. By using timeharmonic domain, the equations can be simplified using only one element of a vector function. Consecutively, the wave equation, which is described a wave propagated along the $z$ direction in Cartesian coordinate system, is given

$$
\frac{d^{2} E_{x}}{d x^{2}}+\omega^{2} \mu \varepsilon E_{x},
$$

where $\omega$ is an angle frequency [rad/s], $\mu$ is a permeability $[\mathrm{H} / \mathrm{m}]$ and $\varepsilon$ is a permittivity $[\mathrm{F} / \mathrm{m}]$. As it can be seen, this equation is without right side that means there are no sources. In general (for all material types), the permittivity is considered as a complex number where the real part is the permittivity of a material and the imaginary part consists of two elements, the first one represents the conduction current and the second one describes the polarization losses. The complex permittivity is substituted into the equation (1) and then it can be written

$$
\frac{d^{2} E_{x}}{d x^{2}}-\gamma^{2} E_{x}=0 \text {, where } \gamma^{2}=-\omega^{2} \mu \varepsilon\left(1-\frac{\mathrm{j} \sigma}{\omega \varepsilon}\right) \text {. }
$$

The propagation constant $\gamma$ can then be expressed in a following way

$$
\gamma=\mathrm{j} \omega \sqrt{\mu \varepsilon} \sqrt{\left(1-\frac{\mathrm{j} \sigma}{\omega \varepsilon}\right)}=\alpha+\mathrm{j} \beta,
$$

where $\alpha$ is an attenuation constant $[1 / \mathrm{m}], \beta$ is a phase constant $[\mathrm{rad} / \mathrm{m}]$ and $\sigma$ is an electric conductivity $[\mathrm{S} / \mathrm{m}]$.

The losses are large $(\sigma>>\omega \varepsilon)$ in highly conductive materials and, therefore, the equation (3) can be simplified and it defines the depth of penetration $\delta[\mathrm{m}]$

$$
\delta=\frac{1}{\sqrt{\pi f \mu \sigma}}=\frac{1}{\alpha}=\frac{1}{\beta},
$$

where $f$ is a frequency $[\mathrm{Hz}]$. Equation

$$
E_{x}(z)=E_{0}^{+} e^{-\gamma}=E_{0}^{+} e^{-z / \delta} e^{-\mathrm{j} z / \delta}
$$

is a solution of the wave equation (2) for a propagating wave along the positive $z$ direction. It is possible to write an equation for the $x$ component of current density $J_{x}$ in the similar way. It means that electromagnetic wave or induced eddy currents are attenuated in the highly conductive material by $e^{-\alpha z}$ and the phase lag is changed by $e^{-\mathrm{j} \beta z}$ (Fig. 1). The wave is attenuated to the $1 / e$ of its original amplitude in the depth $\delta$ inside the material.

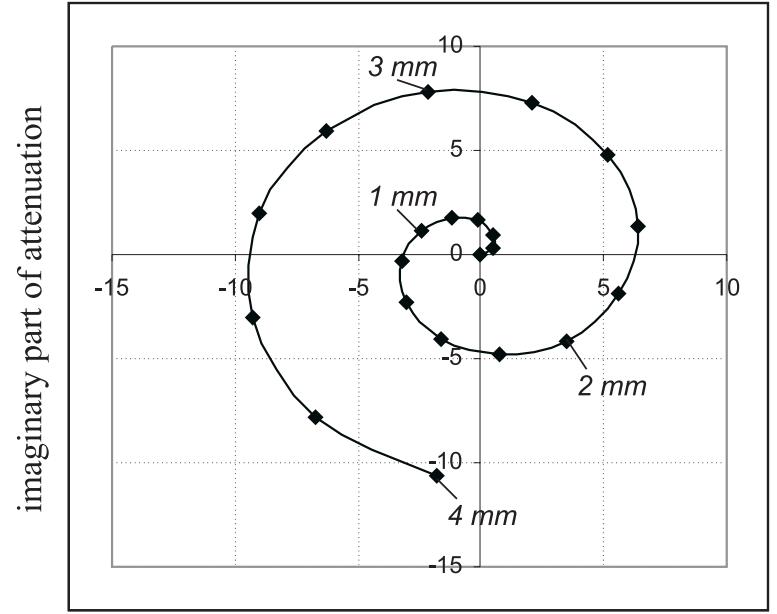

real part of attenuation

Fig. 1 Attenuation spiral of field value in the material with $\delta=0.37 \mathrm{~mm}$

\section{Measurement of frequency influence to the crack detection}

The depth of penetration and hence the rate of the electromagnetic wave attenuation significantly depend on the exciting frequency, the permeability and the conductivity of an inspected material. As the electromagnetic parameters, $\mu$ and $\sigma$, of the inspected material are fixed, it is not possible to vary any of them. Thus, it is necessary to choose the appropriate frequency of the exciting electromagnetic field for the inspection. The influence of variation in the excitation frequency to the field distribution during the inner tube inspection is presented in this part of the paper. An axisymmetric excitation coil is located inside a tube. A defect (crack) is positioned under the exciter on the inner surface of the tube and its depth is changed from 0 to $100 \%$ of a tube wall thickness in a step of $20 \%$. The total exciting current is $10 \mathrm{~A}$. The electromagnetic properties of a tube are defined as $\mu_{r}=1$ and $\sigma=5.8 e 7 \mathrm{~S} / \mathrm{m}$ (pure copper), Fig. 2.



Fig. 2 Simulation model (all descriebed regions are axially symmetrical and defined in cylindric coordinate system)

A 2D axisymmetric program based on the finite element method is used for calculating the electromagnetic field distribution. The simulations are done for frequencies of 1, 2, 4, 8, 16 and $32 \mathrm{kHz}$. The $\varphi$ component of the vector of electrical intensity (in cylindric coordinate system) is calculated in two regions to inter- 
pret the field. The first one is a region of the excitation coil. The second region is a symmetric one to the first region but it is placed on the opposite site of the tube wall (Fig. 2). The results from the simulations are given as the integration of $E_{\varphi}$ in the regions. The mean value of the electrical intensity $E$ can be calculated using equation (6)

$$
E=\frac{1}{S} \int_{S} E_{\varphi} d S
$$

where $S$ is the cross-section of region and its value is $S=$ $=4 e-5 \mathrm{~m}^{2}$ for both the regions. The result from the first region is denoted as E1 and from the second one as $E_{2}$.

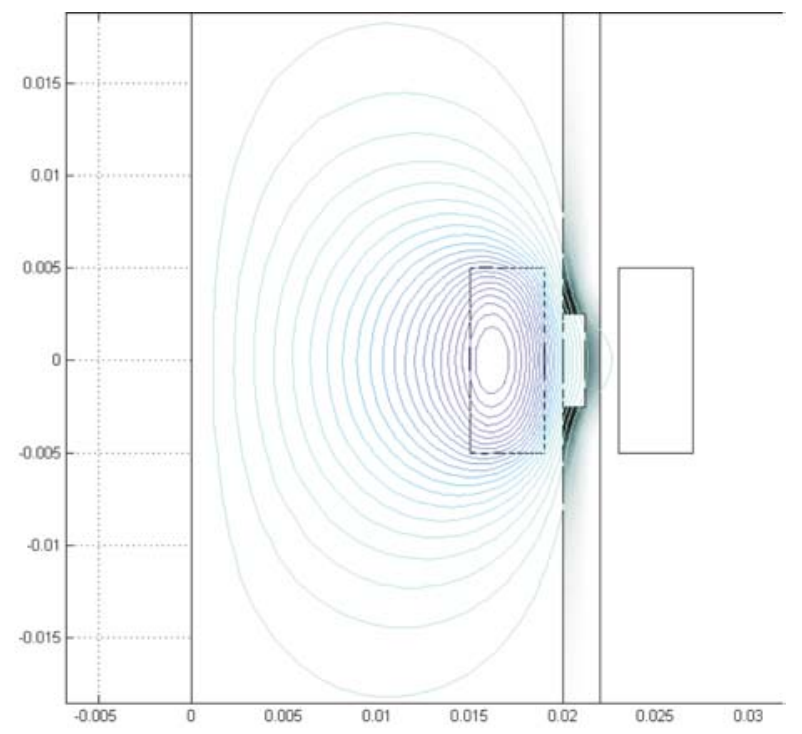

Fig. 3. Absolute values of the electric field intensity (contours) and the induced current density (grey scale, $\max =$ black)

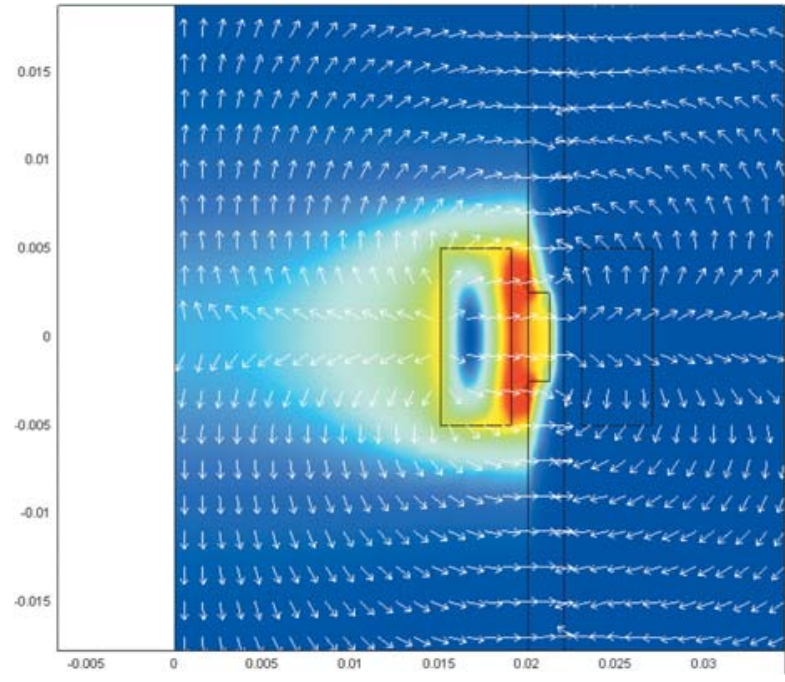

Fig. 4. Absolute values of the magnetic flux density (colour scale, $\max =r e d)$ and the power flow density (normalized arrows)
The simulated components of the electromagnetic field for $f=8 \mathrm{kHz}$ and crack of $60 \%$ in depth can be seen in Figs. 3 and 4. Because the problem is an axisymmetric one it is sufficient to simulate only one half of it.

The relationship between the depth of penetration and the chosen frequencies, counted for the material which is used in simulations (pure copper), shows Fig. 5.

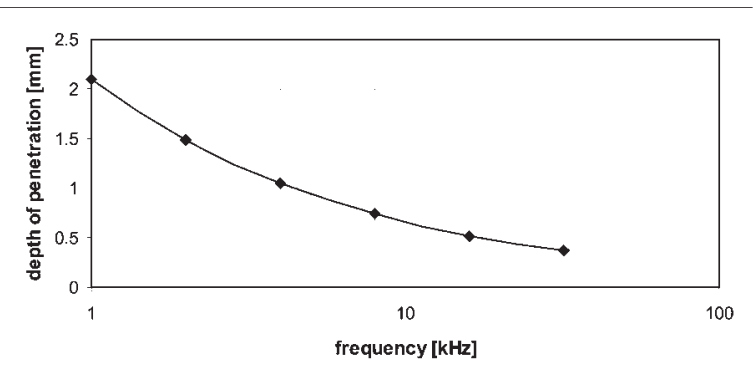

Fig. 5. Relationship between the depth of penetration parameter and the frequency for the constant parameters of material (pure copper)

Fig. 6 shows how the penetration of the field through the tube wall given by $E_{1} / E_{2}$ ratio depends on the depth of the crack. The depth of penetration is only $0.37 \mathrm{~mm}$ for the frequency of $32 \mathrm{kHz}$ which is the reason for the maximal $E_{1} / E_{2}$ ratios difference between $0 \%$ and $100 \%$ cracks. The difference for $1 \mathrm{kHz}$ (where $\delta=2.09 \mathrm{~mm}$ ) is a minimal one.

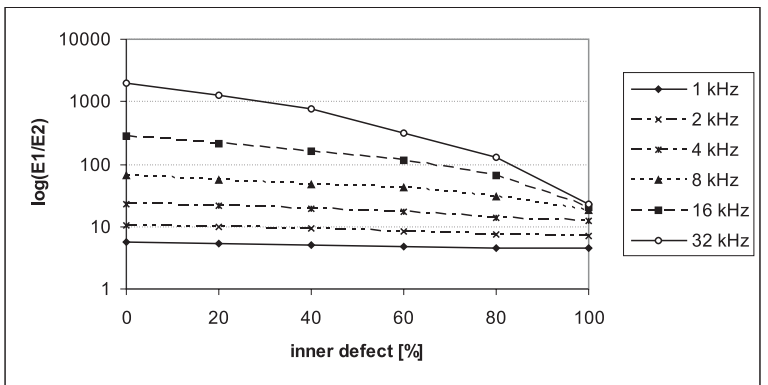

Fig. 6. Rate of penetration of the field throught the tube wall depending up to depth of inner defect (\% of the wall thickness)

In the graphs shown in Figs. 7 and 8 only the results for the first region (inside tube) are shown because that is the side of the exciting coil. The value of $E_{1}$ for $0 \%$ crack is taken as a reference value and this reference is subtracted from the signals of deeper cracks. Therefore, such defined relative values are given in the plots. As it can be seen in Fig. 7, the higher relative values of field intensities are obtained for the higher frequencies that mean better sensitivity to the crack using higher frequencies $(32 \mathrm{kHz})$. On the other hand, Fig. 8 shows that the probe is not very sensitive for deeper cracks recognition using the high frequency $(32 \mathrm{kHz})$ however using the low frequency excitation $(1 \mathrm{kHz})$ it is 
almost equally sensitive to all the depths of the crack, which is an issue of the depth of penetration.

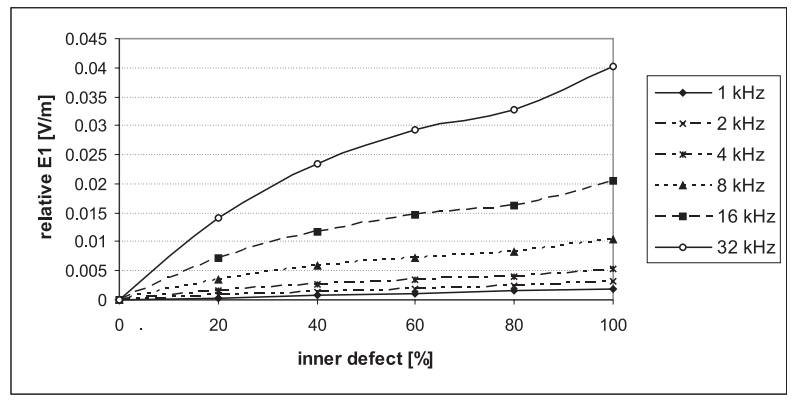

Fig. 7. Relative value of E1

\section{Conclusion}

Based on the simulation results presented in the paper it can be concluded that the selection of the appropriate inspection frequency depends on the material properties as well as on the

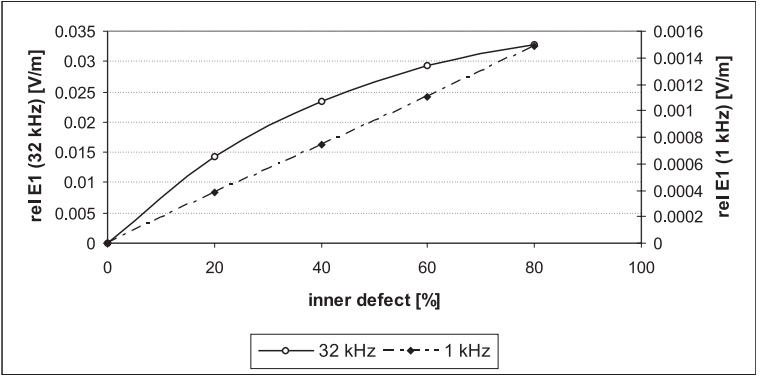

Fig. 8. Relative value of E1 calculated for 1 and $32 \mathrm{kHz}$

expected character of a defect. It is necessary to make a compromise between an intensity of the measured signal (high frequency) and sensitivity to an increasing depth of a defect (low frequency). The depth of penetration can help with this choice and hence it is one of the most important parameters in EC method of NDT. Accordingly, it is possible to allege that the depth of penetration shows limitations of the method e.g. maximum accessible depth of material.

\section{References}

[1] ČÁPOVÁ, K., ČÁP, I., FAKTOROVÁ, D.: Electromagnetic phenomena as the principles of material nondestructive evaluation. In Advances, 3/2004, ISSN 1336-1376, pp. 189-192.

[2] YUSHI SUN: An introduction to electromagnetic nondestructive testing. In KOSE, V., SIEVERT, J. Non-linear electromagnetic systems, ISEM'97, Amsterdam: IOS Press, 1998, ISBN 90-5199-381-1, pp. 145-152.

[3] JANOUSEK, L., YUSA, N., CHEN, Z., MIYA, K.: Recognition of INCONEL weld conductivity variation by means of eddy current testing, In: Studies in Applied Electromagnetics and Mechanics, Vol 24, IOS Press, 2004, pp. 286-293.

[4] NATHAN, I.: Numerical modeling for electromagnetic non-destructive evaluation, London: Chapman \& Hall, 1995, ISBN 0-412-46830-1.

[5] MAYER, D., POLÁK, J.: Methods of solution of electric and magnetic fields (in Czech), Praha: SNTL; Bratislava, ALFA, 1983. 